\title{
Eliminating acetate formation improves citramalate production by metabolically engineered Escherichia coli
}

\author{
Naga Sirisha Parimi, Ian A. Durie, Xianghao Wu, Afaq M. M. Niyas and Mark A. Eiteman*
}

\begin{abstract}
Background: Citramalate, a chemical precursor to the industrially important methacrylic acid (MAA), can be synthesized using Escherichia coli overexpressing citramalate synthase (cimA gene). Deletion of gltA encoding citrate synthase and leuC encoding 3-isopropylmalate dehydratase were critical to achieving high citramalate yields. Acetate is an undesirable by-product potentially formed from pyruvate and acetyl-CoA, the precursors of citramalate during aerobic growth of $E$. coli. This study investigated strategies to minimize acetate and maximize citramalate production in E. coli mutants expressing the cimA gene.
\end{abstract}

Results: Key knockouts that minimized acetate formation included acetate kinase (ackA), phosphotransacetylase (pta), and in particular pyruvate oxidase (poxB). Deletion of glucose 6-phosphate dehydrogenase (zwf) and ATP synthase (atpFH) aimed at improving glycolytic flux negatively impacted cell growth and citramalate accumulation in shake flasks. In a repetitive fed-batch process, E. coli gltA leuC ackA-pta poxB overexpressing cimA generated $54.1 \mathrm{~g} / \mathrm{L}$ citramalate with a yield of $0.64 \mathrm{~g} / \mathrm{g}$ glucose ( $78 \%$ of theoretical maximum yield), and only $1.4 \mathrm{~g} / \mathrm{L}$ acetate in $87 \mathrm{~h}$.

Conclusions: This study identified the gene deletions critical to reducing acetate accumulation during aerobic growth and citramalate production in metabolically engineered E. coli strains. The citramalate yield and final titer relative to acetate at the end of the fed-batch process are the highest reported to date (a mass ratio of citramalate to acetate of nearly 40) without being detrimental to citramalate productivity, significantly improving a potential process for the production of this five-carbon chemical.

Keywords: Acetyl-CoA, Pyruvate, Citramalate, Escherichia coli, Acetate, Glucose, Fed-batch

\section{Background}

Synthetic biology and metabolic engineering have enabled sustainable and eco-friendly manufacturing of commercially important food products, pharmaceuticals, commodity chemicals, and other high value products using microorganisms. Some chemicals which cannot be synthesized exclusively by a biosynthetic route might be generated using hybrid approaches involving both biological and chemical synthesis. For example, methacrylic acid (MAA), a commodity chemical with an estimated annual global market of 2.2 million tons [42] is a monomer of poly(methyl methacrylate) or PMMA, which is

*Correspondence: eiteman@engr.uga.edu

School of Chemical, Materials and Biomedical Engineering, Driftmier

Engineering Center, University of Georgia, Athens, GA 30602, USA used in automobile, construction, medical device, lighting and the home appliance industries. Acrylates in general are very toxic [3], and their direct microbial synthesis at relevant concentrations seems unlikely. MAA can fortunately be synthesized via a hybrid route: biochemical production of citramalate from glucose [40], and subsequently transforming this compound chemically using base-catalyzed decarboxylation and dehydration [22]. Citramalate (or citramalic acid) is naturally found in the metabolic pathways of some anaerobic bacteria $[8,18$, 32].

Escherichia coli is a well-established microbial cell factory for the biotechnology industry. Citramalate production in metabolically engineered $E$. coli expressing citramalate synthase coding the $\operatorname{cim} A$ gene (Fig. 1) has 


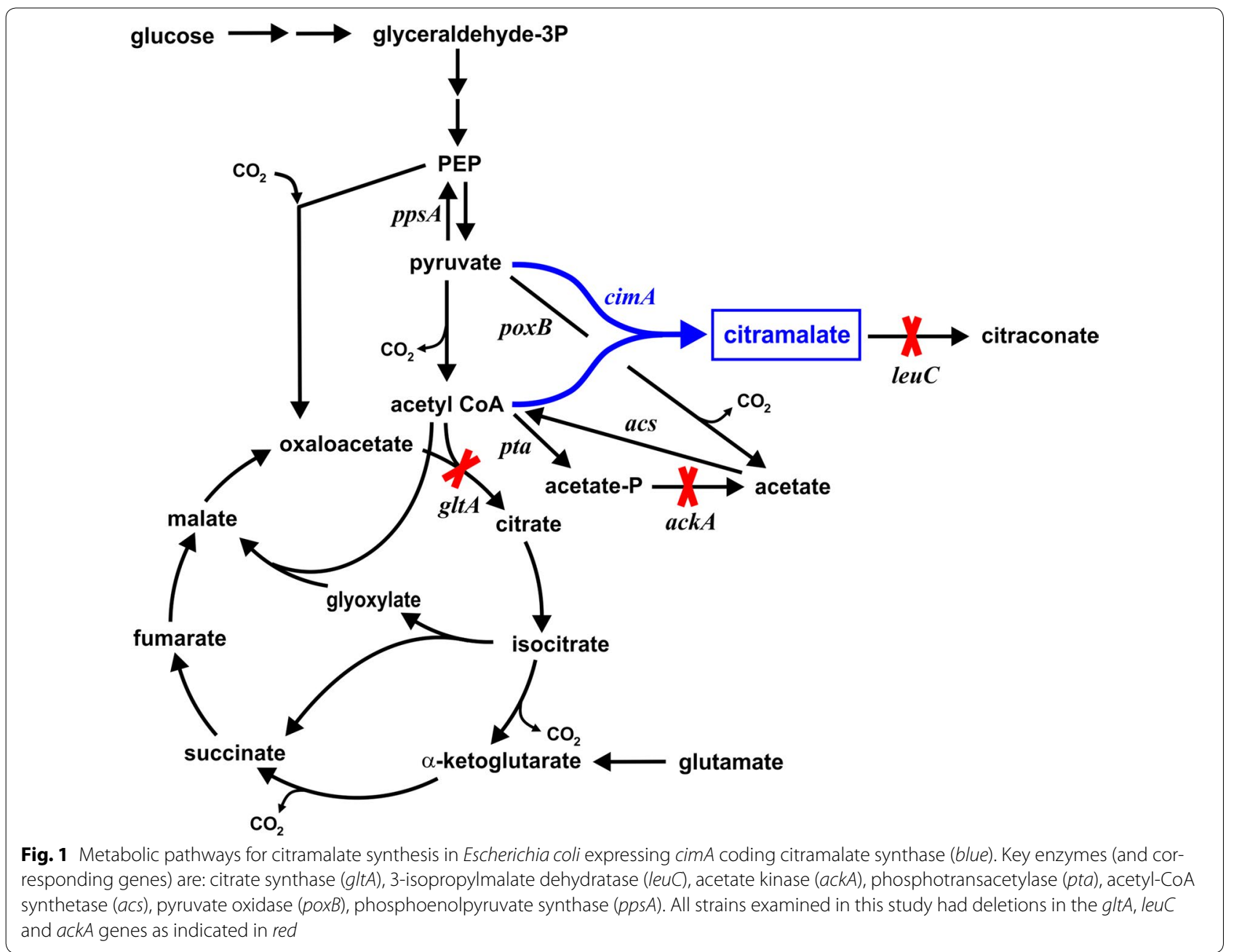

previously been demonstrated $[4,40]$. In a recent study, E. coli MG1655 gltA leuC ackA/pZE12-cimA containing three key knockouts (citrate synthase, 3-isopropylmalate dehydratase and acetate kinase) accumulated $46 \mathrm{~g} / \mathrm{L}$ citramalate from glucose at a yield of $0.63 \mathrm{~g} / \mathrm{g}$ ( $75 \%$ of the theoretical maximum). However, $10 \mathrm{~g} / \mathrm{L}$ acetate also accumulated despite the deletion of acetate kinase. At the end of the fermentation, this by-product accumulated to an ultimate mass ratio citramalate-to-acetate (i.e., desirable product:undesirable product) of less than 5 . Acetate is a typical "overflow" metabolite when wild-type E. coli cells are grown at a high growth rate, and the carbon flux into central metabolic pathways exceeds the cells' biosynthetic demands and the capacity for energy generation [2, 17]. Acetate formation is undesirable because this acid: (i) negatively impacts cell growth even at concentrations as low as $0.5 \mathrm{~g} / \mathrm{L}$ [28]; (ii) is a sink which diverts carbon that could otherwise be used to synthesize the desired product [17]; (iii) necessitates additional downstream separation step(s) that add to process costs. Acetate is generated by two pathways in E. coli: from acetyl-CoA via acetate kinase and phosphotransacetylase (ack $A$ and $p t a$ genes), and from pyruvate via pyruvate oxidase (poxB). While the ackA-pta pathway is typically the route during exponential growth, pyruvate oxidase becomes active during late exponential and early stationary phases [15]. Deletion of $a c k A$ and/or $p t a$ genes has previously resulted in lower growth rates and lower but still significant acetate accumulation in several $E$. coli mutants $[9,11,14$, 15]. Strains with poxB deleted but not the ackA-pta pathway accumulated either similar or slightly lower acetate compared to wild type strains $[15,25]$. Growth of strains in which both acetate producing pathways were deleted showed very low acetate accumulation and growth rates similar to wild type strains $[9,15]$.

The two precursors for citramalate, pyruvate and acetyl-CoA, are generated through the glycolytic pathway, and increasing the flux through glycolysis might improve citramalate productivity and yield. Since the ATP/ADP ratio controls glycolysis [24], previous research has shown that decreasing ATP generation increased the rate of glycolysis [30], and product formation [34, 45]. 
Glycolytic flux may also be improved by preventing flux through the pentose phosphate pathway (PP pathway), for example, by a deletion in glucose 6-phosphate dehydrogenase $(z w f)$ [43].

The goal of this study was to improve the formation of citramalate in E. coli expressing citramalate synthase by blocking acetate formation. We also investigated whether strategies to increase glycolytic flux would increase citramalate yield and productivity.

\section{Results}

\section{Citramalate and acetate formation in shake flasks}

Citramalate synthase (coded by the $\operatorname{cim} A$ gene) mediates the conversion of pyruvate and acetyl-CoA to citramalate. Knockouts in the gltA, leuC and ackA genes coding for citrate synthase, 3-isopropylmalate dehydratase, and acetate kinase, respectively, were critical to achieving high citramalate yield [40]. Despite the deletion of acetate kinase, over $10 \mathrm{~g} / \mathrm{L}$ acetate accumulated in a repetitive fed-batch process [40]. We therefore compared citramalate formation after $24 \mathrm{~h}$ in shake flasks by several E. coli strains having additional gene knockouts and expressing the pZE12-cimA plasmid (Fig. 2). MEC499/ pZE12-cimA ( gltA leuC ackA) serves as the control strain [40]. Specifically, we examined the enzymes involved in acetate formation from acetyl-CoA and pyruvate, the precursors of citramalate. We anticipated that screening at the $50 \mathrm{~mL}$ shake flask scale in shake flasks would provide guidance on strains to study in greater detail at the $1 \mathrm{~L}$ scale in a bioreactor.

In E. coli phosphotransacetylase ( $p t a$ gene) and acetate kinase (ackA) normally produce acetate during the exponential growth phase through the high energy acetyl phosphate (acetyl-P) intermediate [33]. Previous research

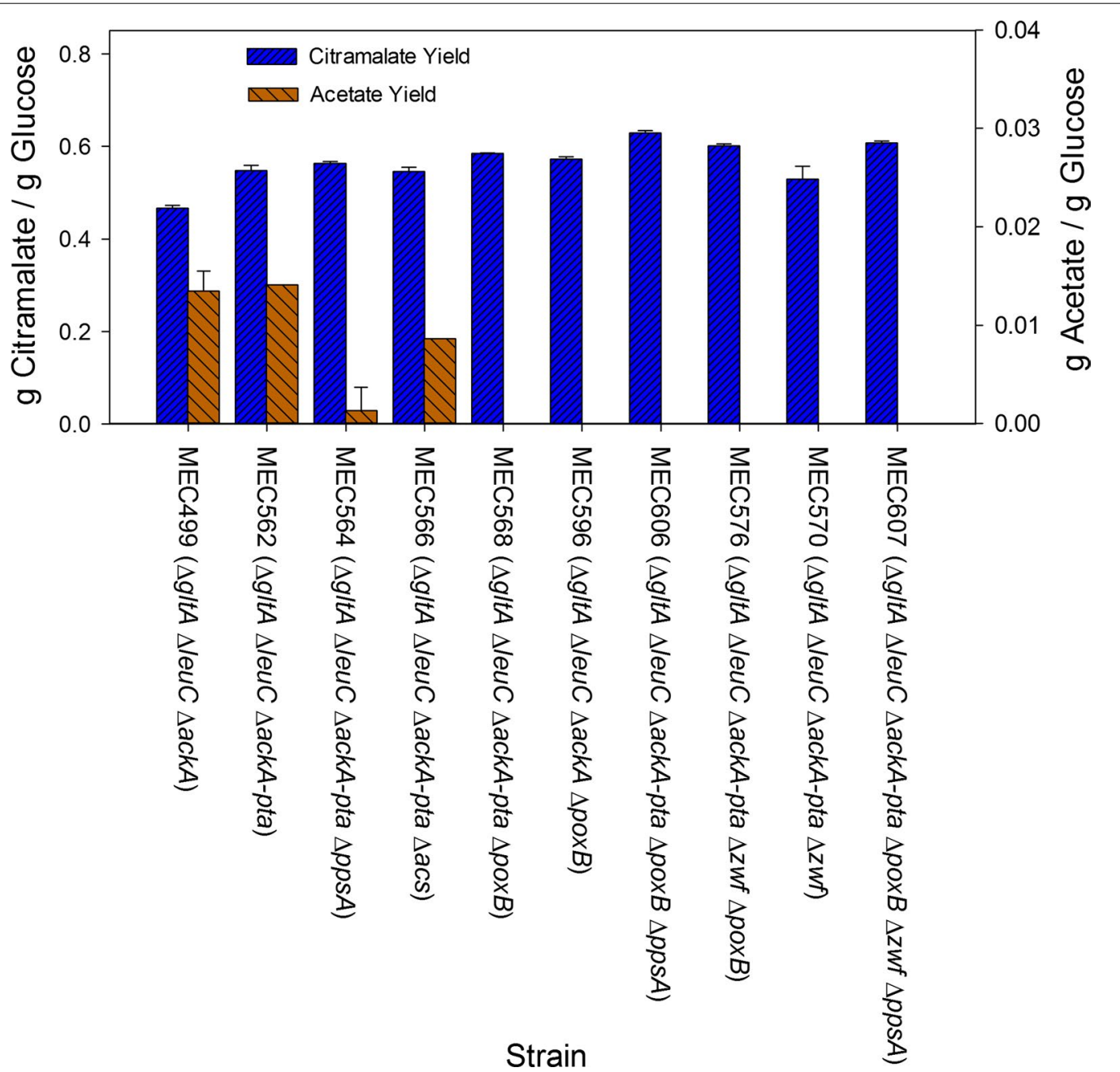

Fig. 2 Comparison of citramalate and acetate yields at $24 \mathrm{~h}$ in shake flasks using various E. coli strains each expressing cimA gene on the pZE12cimA plasmid. The defined medium contained $5 \mathrm{~g} / \mathrm{L}$ glucose, $1 \mathrm{~g} / \mathrm{L}$ L-glutamate and $0.2 \mathrm{~g} / \mathrm{L}$ L-leucine. All studies were carried out in triplicate. Results with MEC499/pZE12-cimA are from [40] 
demonstrated that acetyl-P can form acetate even in the absence of ackA [39]. Since an ackA deletion alone in MEC499 was previously shown to be insufficient to prevent acetate formation [40], we suspected acetyl-P generated via phosphotransacetylase might be responsible for acetate formation. From $5.0 \mathrm{~g} / \mathrm{L}$ glucose, MEC562/ pZE12-cimA ( gltA leuC ackA-pta) attained an OD of 2.70 and accumulated $2.72 \mathrm{~g} / \mathrm{L}$ citramalate and $0.07 \mathrm{~g} / \mathrm{L}$ acetate, similar to the amount of these products observed previously in shake flasks using MG1655 gltA leuC ack $\mathrm{A}$ [40]. Although the combination of pta and ackA did not eliminate acetate formation, MEC562/pZE12-cimA did show a significant increase in citramalate yield compared to MEC499/pZE12-cimA (Fig. 2).

Phosphoenolpyruvate synthase (ppsA) catalyzes the ATP-dependent conversion of pyruvate to phosphoenolpyruvate [6]. A loss of pyruvate through this enzyme could affect citramalate accumulation. However, MEC564/pZE12-cimA (gltA leuC ackA-pta ppsA) generated $2.76 \mathrm{~g} / \mathrm{L}$ citramalate and $0.01 \mathrm{~g} / \mathrm{L}$ acetate. A knockout of phosphoenolpyruvate synthase did not impact citramalate yield compared to MEC562/pZE12-cimA, though surprisingly this knockout reduced acetate formation in shake flasks (Fig. 2).

Acetyl-CoA synthetase (acs) is described as an acetate scavenging enzyme that typically converts acetate to acetyl-CoA [7]. To rule out possible reverse formation of acetate via this enzyme, we constructed MEC566/pZE12$\operatorname{cimA}$ ( gltA leuC ackA-pta acs) containing the additional knockout in acs gene. MEC566/pZE12-cimA generated $1.9 \mathrm{~g} / \mathrm{L}$ citramalate $(0.55 \mathrm{~g} / \mathrm{g}$ yield $)$ and $0.03 \mathrm{~g} / \mathrm{L}$ acetate. Since the three knockouts ackA-pta acs did not eliminate acetate formation (Fig. 2), acetate is likely derived from another metabolite and not acetyl CoA. Moreover, the OD was 30\% lower for MEC566/pZE12-cimA, and not all glucose was consumed at $24 \mathrm{~h}$ compared to MEC562/ pZE12-cimA.

Membrane-bound pyruvate oxidase (poxB) is coupled to the respiratory chain, and oxidizes pyruvate directly to acetate, by-passing acetyl-CoA formation. MEC568/pZE12-cimA ( ltA leuC ackA-pta poxB) generated $2.9 \mathrm{~g} / \mathrm{L}$ citramalate, and no acetate was detected. To address whether pyruvate oxidase or phosphotransacetylase was the more important route to acetate formation, we also examined the performance of the strain retaining the native phosphotransacetylase activity. MEC596/ pZE12-cimA (gltA leuC ackA poxB) generated $2.41 \mathrm{~g} / \mathrm{L}$ citramalate, and no acetate was detected. These results suggest that pyruvate oxidase plays the more important role in acetate formation. MEC596/pZE12-cimA also had 20\% lower $24 \mathrm{~h}$ OD compared to MEC568/ pZE12-cimA. MEC606/pZE12-cimA (gltA leuC ackApta poxB ppsA) accumulated only $2.14 \mathrm{~g} / \mathrm{L}$ citramalate but no acetate, and grew to an OD of 1.92, nearly $30 \%$ lower than MEC562/pZE12-cimA. MEC568/pZE12$\operatorname{cim} A$ showed a significantly increased citramalate yield $(0.58 \mathrm{~g} / \mathrm{g})$ compared to MEC562/pZE12-cimA, while MEC596/pZE12-cimA showed modest increase in citramalate yield $(0.57 \mathrm{~g} / \mathrm{g})$ compared to MEC562/pZE12$\operatorname{cim} A(\mathrm{p}=0.06)$. In summary, the combination of $p t a$ and pox $B$ knockouts appears to be most effective in providing high citramalate yield and preventing acetate formation (Fig. 2). Of course, shake flask results do not necessarily scale to results observed under prolonged conditions in a controlled bioreactor, necessitating additional studies.

Several other strains were examined which were anticipated to benefit citramalate formation, though not affect acetate generation directly. Glucose-6P dehydrogenase (zwf) diverts metabolic flux at glucose-6P from glycolysis into the pentose phosphate pathway, which not only reduces glycolytic flux, but also lowers the yield of pyruvate and acetyl-CoA [43]. To examine the impact of this pathway on citramalate formation, three strains containing the $z w f$ gene deletion were constructed. Each of these strains having the additional $z w f$ deletion showed slightly lower citramalate yields (Fig. 2). However, because they consistently grew much slower than the corresponding strain containing the $z w f$ gene, the final citramalate concentrations were much lower $(1.31-1.74 \mathrm{~g} / \mathrm{L})$.

Previous results have demonstrated that lowering the cellular ATP level increases glycolytic flux [24, 30], increases ethanol yield in yeast [34], and increases pyruvate yield in recombinant $E$. coli [45]. However, MEC638/ pZE12-cimA (gltA leuC ackA-pta poxB atpFH) was unable to grow in the glucose/glutamate/leucine defined medium.

In summary, the poxB knockout was shown to be important in reducing acetate accumulation in a strain having gltA leuC ackA knockouts and expressing citramalate synthase. The $z w f$ knockout significantly reduced growth rate, while the atpFH knockout prevented growth altogether.

\section{Citramalate and acetate formation in bioreactors}

Results from screening strains in shake flasks do not necessarily transfer to a bioreactor which unavoidably operates under different environmental conditions (mixing, oxygenation, $\mathrm{pH}$ control, etc.). We therefore selected a few strains based on encouraging shake flask results for studies at the larger scale. The poxB knockout appeared important for the elimination of acetate, while several other single or combinations of gene deletions severely reduced growth. To confirm the importance of $p o x B$ and more carefully observe differences between key gene knockouts, we selected for controlled batch studies the experimental control strain MEC499/pZE12-cimA ( gltA 
leuC ack $A)$, and also MEC562/pZE12-cimA (gltA leuC ackA-pta), MEC568/pZE12-cimA (gltA leuC ackA-pta poxB), MEC596/pZE12-cimA (gltA leuC ackA poxB) and MEC606/pZE12-cimA (gltA leuC ackA-pta poxB ppsA).

In controlled batch experiments using nominally $30 \mathrm{~g} / \mathrm{L}$ glucose, we compared these five strains for citramalate yield, acetate yield and volumetric productivity of citramalate (Table 1). As a "control strain", MEC499/pZE12$\operatorname{cim} A$ reached an OD of 13 in $72 \mathrm{~h}$, and accumulated $19.1 \mathrm{~g} / \mathrm{L}( \pm 0.2)$ citramalate and $1.0 \mathrm{~g} / \mathrm{L}( \pm 0.2)$. This strain generated citramalate at the slowest rate of all the strains examined. MEC596/pZE12-cimA and MEC606/pZE12$\operatorname{cim} A$ showed modestly greater citramalate yield than MEC499/pZE12-cimA ( $\mathrm{p}=0.07)$. MEC596/pZE12-cimA, lacking $p o x B$ but retaining $p t a$, showed the lowest acetate yield, but this result did not correspond with the greatest citramalate yield. The highest (statistically indistinguishable) citramalate yields were attained by MEC562/ pZE12-cimA (Fig. 3) and MEC568/pZE12-cimA (Fig. 4) while MEC568/pZE12-cimA resulted in lower acetate yield than all other strains except MEC596/pZE12-cimA. MEC568/pZE12-cimA also showed significantly higher citramalate productivity than all other strains, demonstrating that low acetate formation is accompanied by faster acetyl CoA conversion to citramalate. Despite the fact that the acetate yield was significantly lower using MEC568/pZE12-cimA (0.015 g/g) compared to either MEC562/pZE12-cimA or MEC606/pZE12-cimA (Fig. 5), the citramalate yield from glucose for each of these three strains was similar at $0.60-0.64 \mathrm{~g} / \mathrm{g}$. Other potential by-products including succinate, lactate, ethanol, and pyruvate were not detected $(<0.02 \mathrm{~g} / \mathrm{L})$. The citramalate synthase activity was 31-40 IU/g DCW during the exponential phase for each of the strains.

Strains containing the poxB gene deletion (MEC596/ pZE12-cimA and MEC568/pZE12-cimA) generated the least acetate in batch experiments, while the additional ppsA knockout (MEC606/pZE12-cimA) actually increased acetate formation. Because MEC568/pZE12$\operatorname{cim} A$ also had the highest rate of citramalate generation, we chose this strain for a repetitive fed-batch process.

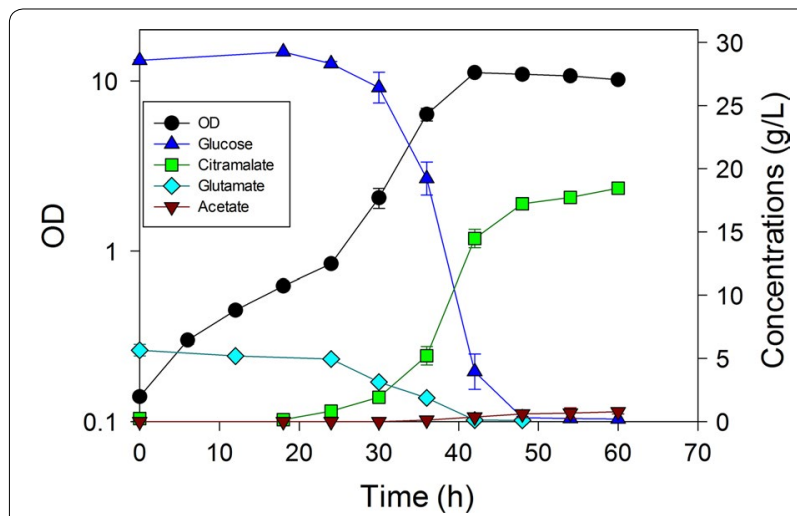

Fig. 3 Time course of citramalate production by E. coli MEC562/ pZE12-cimA ( $\triangle g \mid t A \triangle l e u C \triangle a c k A-p t a)$ in duplicate batch culture. The defined medium contained $30 \mathrm{~g} / \mathrm{L}$ glucose, $5 \mathrm{~g} / \mathrm{L}$ L-glutamate and $1.0 \mathrm{~g} / \mathrm{L}$ L-leucine

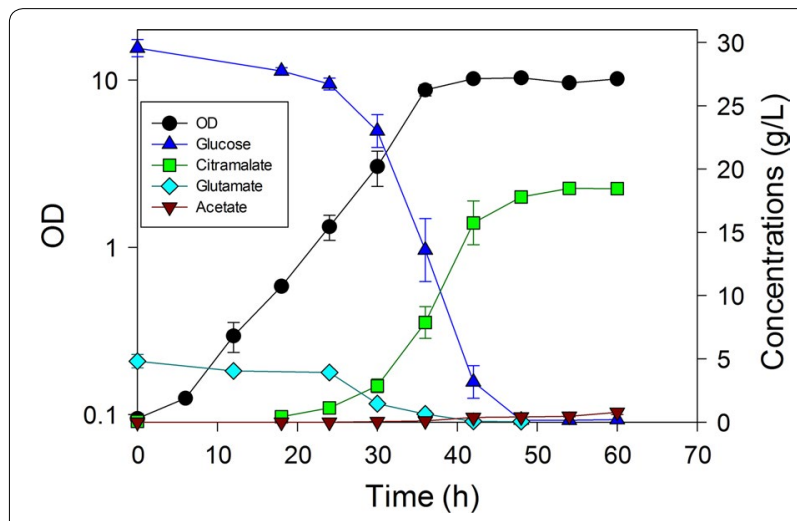

Fig. 4 Time course of citramalate production by E. coli MEC568/ pZE12-cimA ( $\triangle$ gltA $\triangle$ leuC $\triangle a c k A$-pta $\triangle p o x B)$ in duplicate batch culture. The defined medium contained $30 \mathrm{~g} / \mathrm{L}$ glucose, $5 \mathrm{~g} / \mathrm{L}$ L-glutamate and $1.0 \mathrm{~g} / \mathrm{L}$ L-leucine

Specifically, the process commenced as a batch process, and the glucose concentration was monitored. When the glucose concentration decreased to below $5 \mathrm{~g} / \mathrm{L}$, an additional $20 \mathrm{~g}$ glucose, $5 \mathrm{~g}$ L-glutamate and $1 \mathrm{~g}$ L-leucine were added. This batch-wise nutrient feed was accomplished four times during the course of the study, and the

Table 1 Batch fermentations with strains of $E$. coli having knockouts in genes associated with acetate formation and expressing citramalate synthase via the PZE12-cimA plasmid

\begin{tabular}{|c|c|c|c|c|}
\hline Strain & Key gene deletions & Citramalate yield $(\mathrm{g} / \mathrm{g})$ & Acetate yield (g/g) & Volumetric productivity $(g / L h)$ \\
\hline MEC499/pZE12-cimA & gltA leuC ackA & $0.585^{\mathrm{a}}$ & $0.030^{\mathrm{a}}$ & $0.27^{\mathrm{a}}$ \\
\hline MEC562/pZE12-cimA & gltA leuC ackA-pta & $0.642^{b}$ & $0.027^{\mathrm{a}}$ & $0.31^{b}$ \\
\hline MEC596/pZE12-cimA & gltA leuC ackA poxB & $0.609^{\mathrm{a}}$ & $0.007^{c}$ & $0.33^{c}$ \\
\hline MEC568/pZE12-cimA & gltA leuC ackA-pta poxB & $0.626^{a, b}$ & $0.015^{b}$ & $0.34^{d}$ \\
\hline MEC606/pZE12-cimA & gltA leuC ackA-pta poxB ppsA & $0.604^{\mathrm{a}}$ & $0.023^{\mathrm{a}}$ & $0.33^{c}$ \\
\hline
\end{tabular}

The media for all experiments contained initially $30 \mathrm{~g} / \mathrm{L}$ glucose, $5 \mathrm{~g} / \mathrm{L}$ glutamate and $1 \mathrm{~g} / \mathrm{L}$ leucine. Values in a column with different letters indicate significant difference at $p<0.05$ 


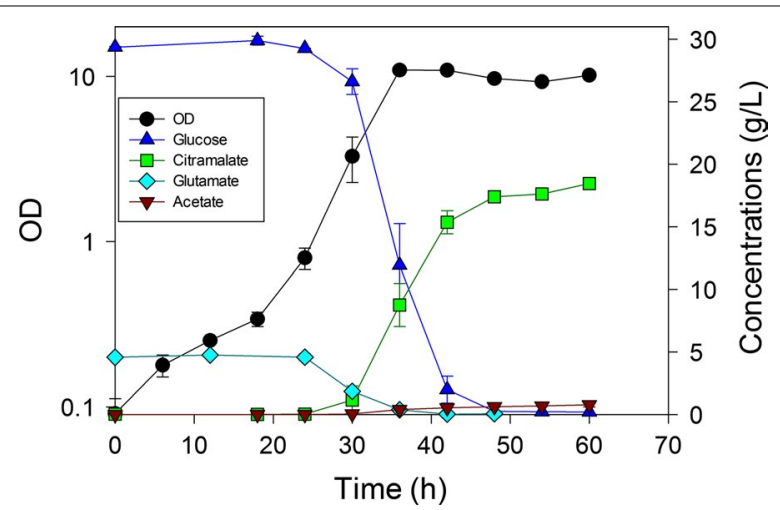

Fig. 5 Time course of citramalate production by E. coli MEC606/ pZE12-cimA ( $\triangle$ gltA $\triangle l e u C \triangle a c k A-p t a \triangle p o x B \triangle p p s A)$ in duplicate batch culture. The defined medium contained $30 \mathrm{~g} / \mathrm{L}$ glucose, $5 \mathrm{~g} / \mathrm{L}$ L-glutamate and $1.0 \mathrm{~g} / \mathrm{L} \mathrm{L-leucine}$

OD achieved by the cells after $87 \mathrm{~h}$ was 20.5 (Fig. 6). At this time the citramalate concentration was $54.1 \mathrm{~g} / \mathrm{L}$, and the yield on glucose was $0.64 \mathrm{~g} / \mathrm{g}$, while the acetate concentration was only $1.4 \mathrm{~g} / \mathrm{L}(0.016 \mathrm{~g} / \mathrm{g}$ yield $)$. Citramalate synthase activity decreased from $35 \mathrm{IU} / \mathrm{g}$ DCW at $39 \mathrm{~h}$ to $12 \mathrm{IU} / \mathrm{g} \mathrm{DCW}$ at $87 \mathrm{~h}$. The final mass ratio of citramalate to acetate was approximately 39 , and the overall citramalate productivity was $0.62 \mathrm{~g} / \mathrm{L} \mathrm{h}$, both the highest reported to date.

\section{Discussion}

In this study, citramalate at a high final concentration $(54.1 \mathrm{~g} / \mathrm{L})$ and yield $(0.64 \mathrm{~g} / \mathrm{g})$ was formed in an $E$. coli cell factory overexpressing citramalate synthase $(\operatorname{cim} A)$ gene. We observed over $85 \%$ less acetate and a greater citramalate yield compared to a previous study [40]. This

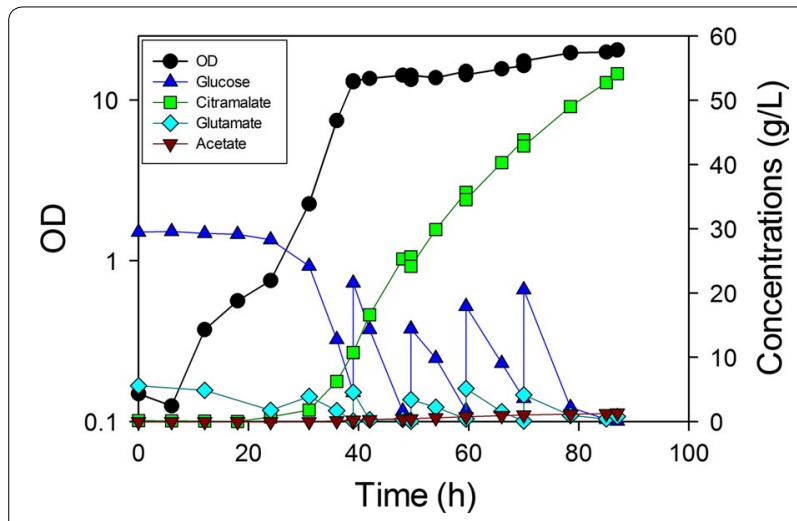

Fig. 6 Time course of citramalate production by E. coli MEC568/ pZE12-cimA ( $\triangle$ gltA $\triangle$ leuC $\triangle a c k A-p t a \triangle p o x B)$ in fed-batch culture. The defined medium initially contained $30 \mathrm{~g} / \mathrm{L}$ glucose, $5 \mathrm{~g} / \mathrm{L}$ L-glutamate and $1.0 \mathrm{~g} / \mathrm{L}$ L-leucine. When the glucose concentration decreased below $5 \mathrm{~g} / \mathrm{L}, 20.0 \mathrm{~g}$ glucose, $5.0 \mathrm{~g}$ L-glutamate and $1.0 \mathrm{~g}$ L-leucine dissolved together in $35 \mathrm{~mL}$ DI water were added four times reduction in acetate accumulation was accomplished by knocking out the ackA-pta and poxB genes, coding for the two major acetate production pathways in $E$. coli. Some acetate (less than $2 \mathrm{~g} / \mathrm{L}$ ) was still observed during batch and fed-batch fermentation processes in the strains containing ackA-pta and poxB gene deletions (MEC568/ pZE12-cimA and MEC606/pZE12-cimA). In a previous study, only $1.7 \mathrm{~g} / \mathrm{L}$ acetate from $40 \mathrm{~g} / \mathrm{L}$ glucose was similarly reported in an ackA-pta and poxB triple mutant $E$. coli strain [31]. In all cases, most acetate accumulation in these triple knockouts occurred in the late exponential and stationary phases. This observation suggests that acetate formation is activated in ackA-pta poxB strains only when cells are under stress during late exponential and stationary phases, perhaps when a portion of the cellular components are being degraded. Although poxB is regulated by the sigma factor encoded by $\operatorname{rpoS}[10]$ and is preferentially used at low growth rates [1], it is unclear how acetate forms in this triple knockout. Moreover, previous studies reporting acetate formation in ackApta poxB strains, in contrast to the present study, used a complex (LB) medium with glucose $[15,31]$. Our study using defined medium suggests that acetate is indeed formed from glucose rather than from an unknown component in a complex medium. Many catabolic reactions generate acetate, and some anabolic pathways including $N$-acetylglucosamine-6-phosphate deacetylase (nagA gene), UDP-3-O-acyl- $N$-acetylglucosamine deacetylase $(l p x C)$, acetylornithine deacetylase $(\operatorname{argE})$, cysteine synthases (cysM and cysK), and acetoacetyl-CoA transferases (ato $A$ and atoD) pathways might contribute to acetate accumulation [31].

One strategy proposed to reduce acetate accumulation is the overexpression of the acetate scavenging acetylCoA synthetase (acs) [26]. This pathway helps accumulate acetyl-CoA and hence could benefit citramalate production. Since the saturation of respiratory capacity and resultant increase in the $\mathrm{NADH} / \mathrm{NAD}+$ ratio are also known to drive metabolism towards acetate generation $[13,37]$, efforts to decrease NADH generation may prove useful. For example, expression of NADH oxidase in an E. coli $\operatorname{arcA}$ mutant eliminated acetate formation at high growth rates [37, 38]. Nevertheless, the complete elimination of acetate while achieving high yield for another product is a challenging problem because it requires a multigene approach and detailed attention to futile pathways, anaplerotic pathways, precursor levels, coenzyme levels, and redox ratios [13]. A comprehensive understanding of the impact of genetic interventions on the metabolic flux distribution through modeling and flux analysis might help fine tune these efforts.

We speculated that knocking out ackA-pta poxB might result in a greater intracellular accumulation of 
pyruvate and acetyl-CoA, the precursors of citramalate and hence improve the yield of this biochemical from glucose, though the yield was indistinguishable from the yield previously reported for MG1655 gltA leuC ackA expressing citramalate synthase [40]. Glycolysis and the PP pathway are the two major glucose catabolic pathways in E. coli, and NADH accumulation during glycolysis induces acetate formation via pyruvate oxidase [37]. Previously, poxB mutants have been observed to increase carbon flux through PP pathway by upregulating glucose 6-phosphate dehydrogenase [25]. In this study, the effort to decrease acetate formation by a knockout in the poxB gene may have led to the partial redirection of glucose into the PP pathway instead of glycolysis. If such a redirection occurred, any potential improvement in citramalate yield through increased availability of acetyl CoA might be compensated by the loss in yield resulting from the elevated PP pathway flux.

The PP pathway protects cells against oxidative stress by generating reducing equivalents as NADPH [20]. $E$. coli strains blocked in the PP pathway, for example, by deleting the $z w f$ gene, compensate for the loss in NADPH formation by increasing glucose uptake rate, increasing the activity of isocitrate dehydrogenase and increasing the TCA cycle flux $[29,43]$. Thus, a $z w f$ knockout may improve yields of products whose biosynthetic pathways involve glycolysis or TCA cycle metabolites. For instance, $z w f$ gene deletion enhanced lycopene production by over $130 \%$ in recombinant $E$. coli strains, owing to an improved Emden-Meyerhof-Parnas (EMP) pathway flux and increased pyruvate [44]. Similarly, a 4.4-fold increase in yield was reported in an E. coli tpiA $z w f$ strain generating 3-hydroxypropionic acid from glycerol [35]. In our shake flask studies, strains with $z w f$ deletions resulted in much lower growth and citramalate accumulation. Unlike previous studies using $z w f$ strains, though, the strains in this study also contained a gltA knockout that prevented carbon flow from acetyl CoA into the TCA cycle, and glutamate was supplied as a secondary carbon source. Thus, the cells were unable to respond to a block in the PP pathway by generating NADPH in the TCA cycle (i.e., isocitrate dehydrogenase), resulting in significantly reduced glucose uptake and growth rate. Growth and citramalate production in a $z w f$ knockout might be improved by engineering another strategy to generate NADPH [27].

We speculated that any intracellular pyruvate accumulation in the gltA strain might result in loss of carbon through PEP synthase, and preventing this loss by knocking out the ppsA gene could result in pyruvate accumulation. However, no significant benefit of a $p p s A$ knockout on citramalate production was observed in the shake flask or batch reactor studies. Gluconeogenic genes are activated in E. coli during the metabolic switch from glucose to acetate consumption [23]. Since the strains examined in this study exhausted glucose only at the end of the process and generated low concentrations of acetate, such a switch may not have been a factor, making $p p s A$ irrelevant.

ATPase plays a major role in metabolic control, and mutations in ATP synthase increase glycolytic flux [24]. Increased glycolytic flux normally leads to increased acetate excretion through acetate kinase as a means to replenish ATP through substrate level phosphorylation [30]. Growth rate and growth yield are related to the rate of ATP synthesis and the amount of ATP synthesized per unit of substrate consumed [21]. In this study, the strain with the atpFH knockouts (MEC638) was unable to generate acetate as a consequence of the ackA-pta poxB knockouts, and with little metabolic flexibility, failed to grow in the glucose/glutamate/leucine medium.

Potassium hydroxide $(\mathrm{KOH})$ was used for $\mathrm{pH}$ control in the fermentation processes. At the end of the fed-batch process (Fig. 6), the concentration of $\mathrm{K}^{+}$ions estimated from the volume of base added to control the $\mathrm{pH}$ was $1.1 \mathrm{~mol} / \mathrm{L}$, while the $\mathrm{NH}_{4}{ }^{+}$concentration was measured to be $188 \mathrm{mg} / \mathrm{L}$. Previous research has demonstrated that the E. coli growth ceases at a $\mathrm{K}^{+}$concentration of $1.1 \mathrm{~mol} / \mathrm{L}$ [41], so the current process may become limited in citramalate formation as a result of the accumulation of the counter-ion needed for $\mathrm{pH}$ control.

\section{Conclusions}

This study reports citramalate production at high yield with low acetate accumulation in metabolically engineered $E$. coli overexpressing citramalate synthase by a codon-optimized $\operatorname{cim} A$ gene. The key knockouts critical to minimizing acetate formation were identified as $p t a, a c k A$ and $p o x B$. Knockouts of $z w f$ and atpFH genes, targeted at improving citramalate production by increasing the glycolytic flux and rate, did not show promising results in shake flask studies. Future work will be aimed at further exploring other metabolic and process engineering strategies to achieve higher titers of citramalate without requiring glutamate in the medium while eliminating acetate.

\section{Methods}

\section{Strain construction and growth media}

Strains used in this study are listed in Table 2. The P1 phage method was used for transducing gene mutations into E. coli MG1655 from their respective strains in the KEIO collection [5]. When necessary for additional gene deletions, a strain was cured of kanamycin using the pCP20 plasmid [12]. All constructs were confirmed using PCR. All strains were transformed with pZE12-cimA 
Table 2 Strains used in this study

\begin{tabular}{|c|c|c|}
\hline Strains & Genotype & References \\
\hline MEC499 & MG1655 $\triangle g \mid t A 770::(F R T) \triangle l e u C 778::(F R T) \triangle a c k A 778:: K a n$ & [40] \\
\hline MEC562 & MG1655 $\Delta g \mid t A 770::(F R T) \triangle l e u C 778::(F R T) \triangle a c k A 778::($ FRT $) \Delta p t a 779:: K a n$ & This study \\
\hline MEC563 & MG1655 $\Delta g / t A 770::(F R T) \Delta l e u C 778::(F R T) \triangle a c k A 778-p t a 779::(F R T)$ & This study \\
\hline MEC564 & MEC563 $\triangle p p s A 776:$ Kan & This study \\
\hline MEC566 & MEC563 $\triangle a c s-763:: K a n$ & This study \\
\hline MEC568 & MEC563 $\triangle p o x B 772:: K a n$ & This study \\
\hline MEC570 & MEC563 $\Delta z w f 777:: K a n$ & This study \\
\hline MEC576 & MEC563 $\triangle z w f 777::($ FRT) $\triangle p o x B 772::$ Kan & This study \\
\hline MEC596 & MG1655 $\triangle g l t A 770:$ (FRT) $\triangle l e u C 778::($ FRT) $\triangle a c k A 778::(F R T) \triangle p o x B 772:: K a n$ & This study \\
\hline MEC606 & MEC563 $\triangle p o x B 772::(F R T) \triangle p p s A 776:: K a n$ & This study \\
\hline MEC607 & MEC563 $\Delta p o x B 772::(F R T) \triangle z w f 777::($ FRT $) \Delta p p s A 776:: K a n$ & This study \\
\hline MEC638 & MEC563 $\triangle p o x B 772::(F R T) \triangle a t p F H:: K a n$ & This study \\
\hline
\end{tabular}

plasmid to express citramalate synthase [40]. Strains were routinely grown at $37^{\circ} \mathrm{C}$ using Lysogeny Broth (LB). The composition of defined XC medium was (per L): $13.3 \mathrm{~g}$ $\mathrm{KH}_{2} \mathrm{PO}_{4}, 4.0 \mathrm{~g}\left(\mathrm{NH}_{4}\right)_{2} \mathrm{HPO}_{4}, 8.4 \mathrm{mg} \mathrm{Na}$ (EDTA) $2 \mathrm{H}_{2} \mathrm{O}$, $1.2 \mathrm{~g} \mathrm{MgSO}_{4} \cdot 7 \mathrm{H}_{2} \mathrm{O}, 4.5 \mathrm{mg}$ thiamine $\cdot \mathrm{HCl}, 13 \mathrm{mg}$ $\mathrm{Zn}\left(\mathrm{CH}_{3} \mathrm{COO}\right)_{2} \cdot 2 \mathrm{H}_{2} \mathrm{O}, 1.5 \mathrm{mg} \mathrm{CuCl}_{2} \cdot 2 \mathrm{H}_{2} \mathrm{O}, 15.0 \mathrm{mg}$ $\mathrm{MnCl}_{2} \cdot 4 \mathrm{H}_{2} \mathrm{O}, 2.5 \mathrm{mg} \mathrm{CoCl}{ }_{2} \cdot 6 \mathrm{H}_{2} \mathrm{O}, 3.0 \mathrm{mg} \mathrm{H} \mathrm{BO}_{3}, 2.5 \mathrm{mg}$ $\mathrm{Na}_{2} \mathrm{MoO}_{4} \cdot 2 \mathrm{H}_{2} \mathrm{O}, 100 \mathrm{mg} \mathrm{Fe}(\mathrm{III})$ citrate, and $100.0 \mathrm{mg}$ citric acid. Carbon sources were added as detailed below. Additionally, either medium was supplemented with $50.0 \mathrm{mg} / \mathrm{L}$ ampicillin and/or $100.0 \mathrm{mg} / \mathrm{L}$ kanamycin as appropriate.

\section{Shake flask and bioreactor studies}

For shake flask studies, cells were first grown in $3 \mathrm{~mL}$ LB for $12-14 \mathrm{~h}$, and then $0.5 \mathrm{~mL}$ transferred to $50 \mathrm{~mL}$ $\mathrm{XC}$ medium with $5.0 \mathrm{~g} / \mathrm{L}$ glucose, $1.0 \mathrm{~g} / \mathrm{L}$ L-glutamate and $0.2 \mathrm{~g} / \mathrm{L}$ L-leucine in $500 \mathrm{~mL}$ shake flasks. Each culture was induced at the time of inoculation with $0.2 \mathrm{mM}$ IPTG. Cultures grew at $37{ }^{\circ} \mathrm{C}$ and $250 \mathrm{rpm}$ (19 mm pitch) for $24 \mathrm{~h}$. Shake flask studies were replicated three or more times, and statistical analyses were completed using Student's t test (two-tailed, equal variance), and $\mathrm{p}<0.05$ was considered the criterion for significance.

All bioreactor studies were conducted in $2.5 \mathrm{~L}$ bioreactors (Bioflo 2000, New Brunswick Scientific Co., New Brunswick, NJ, USA). Cultures were again grown first in $3 \mathrm{~mL} \mathrm{LB}$, then $50 \mathrm{~mL}$ shake flasks as described above, and which were then used to inoculate $1.0 \mathrm{~L} \mathrm{XC} \mathrm{medium} \mathrm{with}$ $30.0 \mathrm{~g} / \mathrm{L}$ glucose, $5.0 \mathrm{~g} / \mathrm{L}$ L-glutamate and $1.0 \mathrm{~g} / \mathrm{L} \mathrm{L-leu-}$ cine. Each culture was induced at the time of inoculation with $0.2 \mathrm{mM}$ IPTG. Agitation was maintained at $400 \mathrm{rpm}$ and air supplemented with pure oxygen if necessary was sparged at $1.0 \mathrm{~L} / \mathrm{min}$ to maintain the dissolved oxygen above $40 \%$ saturation. The $\mathrm{pH}$ was controlled at $7.0 \mathrm{using}$ $20 \%(\mathrm{w} / \mathrm{v}) \mathrm{KOH}$, and the temperature was maintained at $37^{\circ} \mathrm{C}$. For a fed-batch process, $20.0 \mathrm{~g}$ glucose, $5.0 \mathrm{~g} \mathrm{~L}$-glutamate and $1.0 \mathrm{~g}$ L-leucine dissolved together in $35 \mathrm{~mL}$ DI water was added four times when the glucose concentration in the culture decreased below $5.0 \mathrm{~g} / \mathrm{L}$.

\section{Analytical methods}

Optical density (OD) at $600 \mathrm{~nm}$ was measured using a spectrophotometer (UV-650 spectrophotometer, Beckman Instruments, San Jose, CA, USA). Concentrations of extracellular organic acids were measured using HPLC with Refractive Index detection as described previously [16]. Glutamate concentration was measured using a glutamate assay kit (Sigma-Aldrich Co., St. Louis, MO, USA). Ammonia-nitrogen $\left(\mathrm{NH}_{4}-\mathrm{N}\right)$ was determined by the Feed and Environmental Water Lab (University of Georgia, Athens, GA, USA) using the colorimetric EPA method [36].

Cell-free extracts were prepared according to the following procedure: (i) centrifuge sample at $3300 \times g$ for $10 \mathrm{~min}$ at $4{ }^{\circ} \mathrm{C}$; (ii) wash the cell pellet twice with $100 \mathrm{mM}$ Tris. $\mathrm{HCl}(\mathrm{pH} 8.0)$ at $4{ }^{\circ} \mathrm{C}$; (iii) resuspend in $100 \mathrm{mM}$ Tris. $\mathrm{HCl}(\mathrm{pH} 8.0)$ at $4{ }^{\circ} \mathrm{C}$; (iv) lyse cells using a French ${ }^{\circledR}$ press (Thermospectronic, Rochester, NY, USA) at 14,000 psi with 2-3 passes; (v) remove cell debris by centrifugation at $20,000 \times g$ for $15 \mathrm{~min}$ at $4{ }^{\circ} \mathrm{C}$. Citramalate synthase enzyme activity was measured in the cell-free extracts following a previous protocol [19]. Briefly, the rate of free CoA generated at $37{ }^{\circ} \mathrm{C}$ was determined by detecting its reaction product with $5,5^{\prime}$-dithiobis(2-nitrobenzoic acid) at $412 \mathrm{~nm}$.

\section{Authors' contributions}

NSP, XW and MAE conceived of the study and designed components of the research. NSP, IAD, and AMMN carried out the fermentations and acquired the data. NSP, IAD, AMMN and MAE interpreted the results. NSP prepared the manuscript draft. All authors provided input for the manuscript. All authors read, and approved the final manuscript. 


\section{Acknowledgements}

The authors acknowledge Sarah A. Lee for assistance with analytical measurements.

\section{Competing interests}

The authors declare that they have no competing interests.

\section{Consent for publication}

The authors provide consent for publication.

\section{Availability of data and materials}

The datasets supporting the conclusions of this article are included with the article. Strains examined are available from the corresponding author.

\section{Publisher's Note}

Springer Nature remains neutral with regard to jurisdictional claims in published maps and institutional affiliations.

Received: 21 February 2017 Accepted: 19 June 2017

Published online: 21 June 2017

\section{References}

1. Abdel-Hamid AM, Attwood MM, Guest JR. Pyruvate oxidase contributes to the aerobic growth efficiency of Escherichia coli. Microbiology. 2001:147:1483-98.

2. Akesson M, Karlsson EN, Hagander P, Axelsson JP, Tocaj A. On-line detection of acetate formation in Escherichia coli cultures using dissolved oxygen responses to feed transients. Biotechnol Bioeng. 1999;64:590-8.

3. Arya AS, Lee SA, Eiteman MA. Differential sensitivities of the growth of Escherichia coli to acrylate under aerobic and anaerobic conditions and its effect on product formation. Biotechnol Lett. 2013;35:1839-43.

4. Atsumi S, Liao JC. Directed evolution of Methanococcus jannaschii citramalate synthase for biosynthesis of 1-propanol and 1-butanol by Escherichia coli. Appl Environ Microbiol. 2008;74:7802-8.

5. Baba T, Ara T, Hasegawa M, Takai Y, Okumura Y, Baba M, Datsenko KA, Tomita M, Wanner BL, Mori H. Construction of Escherichia coli K-12 in-frame, singlegene knockout mutants: the Keio collection. Mol Syst Biol. 2006;2:2006.0008.

6. Berman KM, Cohn M. Phosphoenolpyruvate synthetase of Escherichia coli. Purification, some properties, and the role of divalent metal ions. J Biol Chem. 1970;245:5309-18.

7. Brown $\mathrm{T}$, Jones-Mortimer $\mathrm{M}$, Kornberg $\mathrm{H}$. The enzymic interconversion of acetate and acetyl-coenzyme A in Escherichia coli. Microbiology. 1977;102:327-36.

8. Buckel W, Barker H. Two pathways of glutamate fermentation by anaerobic bacteria. J Bacteriol. 1974:117:1248-60.

9. Causey T, Shanmugam K, Yomano L, Ingram L. Engineering Escherichia coli for efficient conversion of glucose to pyruvate. Proc Natl Acad Sci USA. 2004;101:2235-40

10. Chang YY, Wang AY, Cronan JE Jr. Expression of Escherichia coli pyruvate oxidase (PoxB) depends on the sigma factor encoded by the rpoS (katF) gene. Mol Microbiol. 1994;11:1019-28.

11. Contiero J, Beatty C, Kumari S, DeSanti C, Strohl W, Wolfe A. Effects of mutations in acetate metabolism on high-cell-density growth of Escherichia coli. J Ind Microbiol Biotechnol. 2000;24:421-30.

12. Datsenko KA, Wanner BL. One-step inactivation of chromosomal genes in Escherichia coli K-12 using PCR products. Proc Natl Acad Sci. 2000;97:6640-5.

13. De Mey M, De Maeseneire S, Soetaert W, Vandamme E. Minimizing acetate formation in E. coli fermentations. J Ind Microbiol Biotechnol. 2007:34:689-700.

14. Diaz-Ricci J, Regan L, Bailey J. Effect of alteration of the acetic acid synthesis pathway on the fermentation pattern of Escherichia coli. Biotechnol Bioeng. 1991;38:1318-24.

15. Dittrich CR, Vadali RV, Bennett GN, San KY. Redistribution of metabolic fluxes in the central aerobic metabolic pathway of E. coli mutant strains with deletion of the ackA-pta and poxB pathways for the synthesis of isoamyl acetate. Biotechnol Prog. 2005;21:627-31.
16. Eiteman M, Chastain M. Optimization of the ion-exchange analysis of organic acids from fermentation. Anal Chim Acta. 1997;338:69-75.

17. Eiteman MA, Altman E. Overcoming acetate in Escherichia coli recombinant protein fermentations. Trends Biotechnol. 2006;24:530-6.

18. Feng X, Mouttaki H, Lin L, Huang R, Wu B, Hemme CL, He Z, Zhang B, Hicks LM, Xu J. Characterization of the central metabolic pathways in Thermoanaerobacter sp. strain X514 via isotopomer-assisted metabolite analysis. Appl Environ Microbiol. 2009;75:5001-8

19. Howell $\mathrm{DM}, \mathrm{Xu} \mathrm{H}$, White $\mathrm{RH}$. (R)-citramalate synthase in methanogenic archaea. J Bacteriol. 1999;181:331-3.

20. Hua Q, Yang C, Baba T, Mori H, Shimizu K. Responses of the central metabolism in Escherichia coli to phosphoglucose isomerase and glucose6-phosphate dehydrogenase knockouts. J Bacteriol. 2003;185:7053-67.

21. Jensen PR, Michelsen O. Carbon and energy metabolism of atp mutants of Escherichia coli. J Bacteriol. 1992;174:7635-41.

22. Johnson DW, Eastham GR, Poliakoff M, Huddle TA. Method of producing acrylic and methacrylic acid. Google Patents; 2012.

23. Kao KC, Tran LM, Liao JC. A global regulatory role of gluconeogenic genes in Escherichia coli revealed by transcriptome network analysis. J Biol Chem. 2005:280:36079-87.

24. Koebmann BJ, Westerhoff HV, Snoep JL, Nilsson D, Jensen PR. The glycoIytic flux in Escherichia coli is controlled by the demand for ATP. J Bacteriol. 2002;184:3909-16.

25. Li M, Yao S, Shimizu K. Effect of poxB gene knockout on metabolism in Escherichia coli based on growth characteristics and enzyme activities. World J Microbiol Biotechnol. 2007;23:573-80.

26. Lin H, Castro NM, Bennett GN, San K-Y. Acetyl-CoA synthetase overexpression in Escherichia coli demonstrates more efficient acetate assimilation and lower acetate accumulation: a potential tool in metabolic engineering. Appl Microbiol Biotechnol. 2006;71:870-4.

27. Martínez I, Zhu J, Lin H, Bennett GN, San K-Y. Replacing Escherichia coli NAD-dependent glyceraldehyde 3-phosphate dehydrogenase (GAPDH) with a NADP-dependent enzyme from Clostridium acetobutylicum facilitates NADPH dependent pathways. Metab Eng. 2008;10:352-9.

28. Nakano K, Rischke M, Sato S, Märkl H. Influence of acetic acid on the growth of Escherichia coli K12 during high-cell-density cultivation in a dialysis reactor. Appl Microbiol Biotechnol. 1997;48:597-601.

29. Nicolas C, Kiefer P, Letisse F, Körmer J, Massou S, Soucaille P, Wittmann C, Lindley ND, Portais JC. Response of the central metabolism of Escherichia coli to modified expression of the gene encoding the glucose-6-phosphate dehydrogenase. FEBS Lett. 2007;581:3771-6.

30. Noda S, Takezawa Y, Mizutani T, Asakura T, Nishiumi E, Onoe K, Wada M, Tomita F, Matsushita K, Yokota A. Alterations of cellular physiology in Escherichia coli in response to oxidative phosphorylation impaired by defective F1-ATPase. J Bacteriol. 2006;188:6869-76.

31. Phue J-N, Lee SJ, Kaufman JB, Negrete A, Shiloach J. Acetate accumulation through alternative metabolic pathways in ackA- pta- poxB - triple mutant in E. coli B (BL21). Biotechnol Lett. 2010:32:1897-903.

32. Risso C, Van Dien SJ, Orloff A, Lovley DR, Coppi MV. Elucidation of an alternate isoleucine biosynthesis pathway in Geobacter sulfurreducens. J Bacteriol. 2008;190:2266-74.

33. Rose IA, Grunberg-Manago M, Korey SR, Ochoa S. Enzymatic phosphorylation of acetate. J Biol Chem. 1954;211:737-56.

34. Semkiv MV, Dmytruk KV, Abbas CA, Sibirny AA. Increased ethanol accumulation from glucose via reduction of ATP level in a recombinant strain of Saccharomyces cerevisiae overexpressing alkaline phosphatase. BMC Biotechnol. 2014;14:42.

35. Tokuyama K, Ohno S, Yoshikawa K, Hirasawa T, Tanaka S, Furusawa C, Shimizu H. Increased 3-hydroxypropionic acid production from glycerol, by modification of central metabolism in Escherichia coli. Microb Cell Fact. 2014:13:64.

36. U.S. EPA. Nitrogen, ammonia. Method 250.1 (Colorimetric). In: Methods for chemical analysis of water and wastes. EPA-600/4-79-020. Cincinnati: U.S. E. P. A; 1983. p. 350-1.1-350-1.4.

37. Vemuri G, Altman E, Sangurdekar D, Khodursky A, Eiteman M. Overflow metabolism in Escherichia coli during steady-state growth: transcriptional regulation and effect of the redox ratio. Appl Environ Microbiol. 2006;72:3653-61.

38. Vemuri GN, Eiteman MA, Altman E. Increased recombinant protein production in Escherichia coli strains with overexpressed water-forming $\mathrm{NADH}$ oxidase and a deleted ArcA regulatory protein. Biotechnol Bioeng. 2006:94:538-42. 
39. Wanner B, Wilmes-Riesenberg M. Involvement of phosphotransacetylase, acetate kinase, and acetyl phosphate synthesis in control of the phosphate regulon in Escherichia coli. J Bacteriol. 1992;174:2124-30.

40. Wu X, Eiteman MA. Production of citramalate by metabolically engineered Escherichia coli. Biotechnol Bioeng. 2016;113:2670-5.

41. Wu X, Altman R, Eiteman MA, Altman E. Adaptation of Escherichia coli to elevated sodium concentrations increases cation tolerance and enables greater lactic acid formation. Appl Environ Microbiol. 2014;80:2880-8.

42. Zhang K, Woodruff AP, Xiong M, Zhou J, Dhande YK. A synthetic metabolic pathway for production of the platform chemical isobutyric acid. Chemsuschem. 2011:4:1068-70.
43. Zhao J, Baba T, Mori H, Shimizu K. Effect of zwf gene knockout on the metabolism of Escherichia coli grown on glucose or acetate. Metab Eng. $2004 ; 6: 164-74$

44. Zhou Y, Nambou K, Wei L, Cao J, Imanaka T, Hua Q. Lycopene production in recombinant strains of Escherichia coli is improved by knockout of the central carbon metabolism gene coding for glucose-6-phosphate dehydrogenase. Biotechnol Lett. 2013;35:2137-45.

45. Zhu Y, Eiteman MA, Altman R, Altman E. High glycolytic flux improves pyruvate production by a metabolically engineered Escherichia coli strain. Appl Environ Microbiol. 2008;74:6649-55.

\section{Submit your next manuscript to BioMed Central and we will help you at every step:}

- We accept pre-submission inquiries

- Our selector tool helps you to find the most relevant journal

- We provide round the clock customer support

- Convenient online submission

- Thorough peer review

- Inclusion in PubMed and all major indexing services

- Maximum visibility for your research

Submit your manuscript at www biomedcentral com/submit 